\title{
A Retrospective Study of Nine Patients with Progressive Pseudorheumatoid Dysplasia: To Explore Early Diagnosis and Further Treatment
}

\section{Lei Yin ( $\nabla$ yinleik@hotmail.com )}

Shanghai Children's Medical Center Affiliated to Shanghai Jiaotong University School of Medicine https://orcid.org/0000-0003-4795-8710

\section{Youying Mao}

Shanghai Children's Medical Center Affiliated to Shanghai Jiaotong University School of Medicine

\section{Yunfang Zhou}

Shanghai Children's Medical Center Affiliated to Shanghai Jiaotong University School of Medicine

\section{Yongnian shen}

Shanghai Children's Medical Center Affiliated to Shanghai Jiaotong University School of Medicine

\section{Huijin Chen}

Shanghai Childrens Medical Center Affiliated to Shanghai Jiaotong University School of Medicine Department of Hematology and Oncology

\section{Wei Zhou}

Shanghai Children's Medical Center Affiliated to Shanghai Jiaotong University School of Medicine

Yanliang Jin

Shanghai Children's Medical Center Affiliated to Shanghai Jiaotong University School of Medicine Department of Hematology and Oncology

\section{Hua Huang}

Shanghai Children's Medical Center Affiliated to Shanghai Jiaotong University School of Medicine

\section{Yongguo Yu}

Shanghai Jiaotong University School of Medicine Xinhua Hospital

\section{Jian Wang}

Shanghai Childrens Medical Center Affiliated to Shanghai Jiaotong University School of Medicine Department of Hematology and Oncology

\section{Research Article}

Keywords: progressive pseudorheumatoid dysplasia, noninflammatory, articular cartilage, CCN6 gene variant, vitamin D deficiency 
DOI: https://doi.org/10.21203/rs.3.rs-243375/v1

License: (c) (1) This work is licensed under a Creative Commons Attribution 4.0 International License. Read Full License

Version of Record: A version of this preprint was published at Clinical Rheumatology on October 21st, 2021. See the published version at https://doi.org/10.1007/s10067-021-05959-2. 


\section{A retrospective study of nine patients with progressive pseudorheumatoid}

dysplasia: to explore early diagnosis and further treatment

Lei Yin ${ }^{\mathrm{a}, \mathrm{b}, \#, *}$, Youying Mao ${ }^{\mathrm{a}, \#}$, Yunfang Zhou ${ }^{\mathrm{b}}$, Yongnian Shen ${ }^{\mathrm{b}}$, Huijin Chen ${ }^{\mathrm{b}}$, Wei Zhou ${ }^{\mathrm{a}}$, Yanliang Jin ${ }^{\mathrm{c}}$, Hua Huang ${ }^{\mathrm{c}}$, Yongguo Yu ${ }^{\mathrm{d}}$, Jian Wang ${ }^{\mathrm{e}}$,*

a Department of Nephrology, Shanghai Children's Medical Center, Shanghai Jiao tong University School of Medicine, Shanghai, 200127, China

b Special Consultation Clinic for rare and complicated diseases, Shanghai Children's

Medical Center, Shanghai Jiao tong University School of Medicine, Shanghai, 200127,

China

C Department of Rheumatology, Shanghai Children's Medical Center, Shanghai Jiao tong University School of Medicine, Shanghai, 200127, China

${ }^{\mathrm{d}}$ Department of Pediatric Endocrinology, Xinhua hospital, Shanghai Jiao tong University School of Medicine, Shanghai, 200092, China

e Department of Medical Genetics and Molecular Diagnostic Laboratory, Shanghai Children's Medical Center,

Shanghai Jiao tong University School of Medicine, Shanghai, 200127, China

\# The first two authors have contributed equally to this study.

* Corresponding authors

Corresponding authors:

Dr. Lei Yin (yinleik@hotmail.com), Department of Nephrology, Shanghai Children's Medical Center, Shanghai Jiaotong University School of Medicine. 1678 Dongfang Road, Shanghai 200127, P.R China; Fax 86-21-58756923

Dr. Jian Wang (Labwangjian@shsmu.edu.cn), Department of Medical Genetics and Molecular Diagnostic Laboratory, Shanghai Children's Medical Center, Shanghai Jiaotong University School of Medicine. 1678 Dongfang Road, Shanghai 200127, P.R China; Fax 86-21-58756923. 


\section{Conflict of interest}

The authors declare that they have no conflict of interests. 


\section{ABSTRACT}

Background: Most patients with progressive pseudorheumatoid dysplasia (PPRD) are initially misdiagnosed because of disease rarity and lack of awareness by most clinicians. The purpose of this present study was to provide further early diagnostic options and potential treatment to patients with PPRD.

Methods: This was a retrospective study. Clinical manifestations, laboratory test results, radiographic features, Sanger Sequencing to determine CCN6 gene variants, treatment and follow-up records were collected in the patients with PPRD. Time to diagnosis, phenotype and genotype correlation were analyzed.

Results: Nine PPRD children were included. There were 3 pairs of siblings and one patient had parental consanguinity. Five patients were misdiagnosed as juvenile idiopathic arthritis (JIA). The onset of disease in 8 patients was between 3 to 6 years of age. The interval from onset of symptoms to obtaining the diagnosis for 8 of the patients varied from 3.6 years to 20 years. The onset of symptoms included enlarged and stiff interphalangeal joints of the fingers, gait disturbance or joint pain. Laboratory tests revealed normal range of inflammatory parameters. Serum levels of 25-hydroxyvitamin D3 in six patients were below the normal range. Radiographic findings included different degree of abnormal vertebral bodies, epiphyseal enlargement of the interphalangeal joints with juxta-articular osteopenia, or cyst-like structures femoral head. All the patients harbored CCN6 variants, and a total of 7 variants were identified. After the treatment of calcitriol in 5 patients with low level of 25-hydroxyvitamin D3 for 1.25 years to 1.75 years, two of them kept stable, while 3 of them improved gradually.

Conclusions: Combining the patient's family history, clinical features presenting with abnormal gait or enlarged and stiff interphalangeal joints of the fingers, normal inflammatory markers, and the characteristic radiographic findings, we can obtain the clinical diagnosis of PPRD for the patients at a very early stage of the disease. Anterior blunt of the vertebral bodies could be an early radiological sign in the patient even without obvious clinical symptoms and characteristics yet. The patients with PPRD having c.624dupA variant in CCN6 may have delayed onset. Underlying vitamin D 
deficiency should be sought and corrected in patients with PPRD.

Keywords: progressive pseudorheumatoid dysplasia; noninflammatory; articular cartilage; CCN6 gene variant; vitamin D deficiency 


\section{Introduction}

Progressive pseudorheumatoid dysplasia (PPRD) (MIM 208230) is a rare genetic disease initially described by Wynne-Davies et al in $1982^{1}$. This autosomal recessive inherited skeletal dysplasia is characterized by progressive noninflammatory arthropathy affecting primarily the articular cartilage ${ }^{2}$. The prevalence of PPRD has been estimated at one per million in the United Kingdom ${ }^{1}$, but it may be underdiagnosed due to the overlap of clinical and imaging features with other pediatric musculoskeletal disorders, such as juvenile idiopathic arthritis (JIA), Scheuermann's disease, spondyloepiphyseal dysplasia, Stickler syndrome, and mucopolysaccharidosis, especially for $\mathrm{JIA}^{3-6}$. The onset of symptoms is usually in childhood between 3 to 6 years of age, and symptoms progressively worsen with time ${ }^{2}$. As the disease is so rare and clinicians are thus lacking awareness, most patients are initially misdiagnosed ${ }^{7}$. The average delay in diagnosis is 6 to 13 years $^{2}$. The patients are most often referred to a pediatrician, rheumatologist, orthopedic surgeon, and very few to a medical geneticist ${ }^{8}$. We hope to find further clues to aid earlier and correct diagnosis based on the information between the clinical manifestations and genotypes of the patients with PPRD. PPRD is caused by variation in the CCN6 gene which maps to chromosome $6 \mathrm{q} 22$, and encodes a protein in the connective tissue growth factor that is expressed in synoviocytes and chondrocytes involved in bone and cartilage growth and development ${ }^{9}$. Homozygous or compound heterozygous CCN6 variants cause the loss of articular cartilage, which leads to the progressive narrowing of all articular spaces and reduced joint mobility ${ }^{10}$. While this molecular basis was elucidated over 10 years ago, no progress has been made towards a specific treatment for PPRD ${ }^{2}$. Better understanding of the pathology of PPRD may facilitate the PPRD clinical treatment. It was found that chondrocytes from PPRD articular cartilage (AC) tissues are significantly stiffer than those from normal AC articular cartilage. The dense stiffen AC tissue is unable to effectively absorb the impact energy as normal AC and cause the pain of patient. The higher stress inside denser AC might cause the morphology change and even the apoptosis of chondrocytes ${ }^{11} \cdot 1,25(\mathrm{OH})_{2} \mathrm{D}_{3}$ (calcitriol), which is used to treat hypocalcemia, osteoporosis, and prevention of corticosteroid-induced 
osteoporosis $^{12}$. It accelerates chondrocyte apoptosis and improve physical function in rats with RA by the inhibition of NF- $\mathrm{BB}$ signal pathway ${ }^{13}$ and showed chondroprotective effects in human chondrocytes ${ }^{14}$. We suspected that calcitriol may maintain the normal proliferation of articular chondrocytes by accelerating chondrocyte apoptosis. In the present study, we systematically evaluated the clinical history and characteristics, laboratory test results, radiological data and CCN6 gene variants in 9 patients with PPRD recruited by our special consultation clinic, and evaluated the effects of calcitriol in PPRD patients with vitamin D deficiency.

\section{Materials and Methods}

Patients

Research subjects' blood samples and pedigree data were obtained from August 2011 to December 2019 from individuals with a genetic confirmed diagnosis of progressive pseudorheumatoid dysplasia in our center. According to the principles of the declaration of Helsinki, informed written consent was obtained from the patients and their parents. This study was approved by the Ethics Committee of the Shanghai Children's Medical Center, Shanghai Jiao tong University School of Medicine, Shanghai, China. The medical history, results of physical examination, other relevant laboratory investigations and radiological data were obtained from the patients during clinical visits/routine clinical care and systematically analyzed. Venous blood samples were collected from both the patients and their parents.

\section{Laboratory investigations}

Laboratory tests of inflammatory parameters were performed including complete blood count, and C-reactive protein (CRP), erythrocyte sedimentation rate (ESR) and the tests to exclude other diseases including liver function test, serum blood urea and creatinine, rheumatoid factor, antinuclear antibody, HLA-B27, electrolytes, thyroid function tests, parathyroid hormone. And serum level of 25-hydroxyvitamin D3 were performed in some of the patients. 


\section{Sanger Sequencing}

Genomic DNA was extracted from 2-mL peripheral blood samples using the QIAamp Blood DNA Mini Kit ${ }^{\circledR}$ (Qiagen, Hilden, Germany) according to the manufacturer's instructions. All 5 coding exons and the exon-intron boundaries of the CCN6 gene (NM_003880.3) were amplified by polymerase chain reaction (PCR) using the primers described preciously ${ }^{15}$. Sanger sequencing was performed on an Applied Biosystems ABI3730XL sequencer (Thermo Fisher Scientific, Waltham, MA, USA), and the data were then analyzed using MutationSurveyor v.4.0.4 software (SoftGenetics LLC, State College, PA, USA).

In silico analysis of the missense variants

The functional prediction of the identified missense variants was analyzed by SIFT online software (https://sift.bii.a-star.edu.sg/), PolyPhen-2 online software (http://genetics.bwh.harvard.edu/pph2/), and CADD online software (https://cadd.gs.washington.edu/).

\section{Results (table1)}

Nine patients (two males and seven females, seven children and two adults) were referred to our center for diagnostic assistance by local hospitals from 4 provinces of China during the period of August 2011 to December 2019. Five of them (case 3, 4, 6, $7,9)$ were initially diagnosed with juvenile idiopathic arthritis (JIA). Case 9 had been treated with methotrexate and cyclophosphamide for 3 months without improvement. Case 5 was diagnosed with ankylosing spondylitis because of positive HLA-B27. Case 8 had the suspected diagnosis of congenital spondyloepiphyseal dysplasia. Six patients (case 1 and 2, case 3 and 4, case 6 and 7) were 3 pairs of siblings. Case 5 had parental consanguinity. After review of the family histories, clinical symptoms, and laboratory investigations of the patients, there were several clues which guided us to the diagnosis of PPRD. Key indicators were enlargement of the interphalangeal joints of the fingers and varying degree of limitation of joint movement, the lack of increased inflammatory 
parameters (ESR and CRP) in the blood (table1) and the radiological findings of the abnormal vertebral bodies. The patients and their parents were subjected to DNA sequencing of $C C N 6$ to investigate the genetic cause of this anomaly and for appropriate genetic counseling.

History of diagnosis and clinical findings

Eight of the nine patients had onset of symptoms in childhood between 3 and 6 years of age. Only case 8 began to have the symptoms from 14 years old. The interval between onset of symptoms and obtaining the diagnosis with PPRD for all patients except case 2 varied between 3.6 years to 20 years. Case 2 got the definite diagnosis in one month without obvious joint enlargement or movement limitation (Figure1a, 1b) since the patient's older brother (case1) had already been diagnosed with PPRD. The onset of symptoms for the observed patients included enlarged and stiff interphalangeal joints of the fingers (2/9), gait disturbance (5/9) or joint pain (4/9). Causes for clinic-visits were the worsening of symptoms, most (7/9) had progressive abnormal gait, one patient (case 1) had short stature, and one patient (case 2) visited the clinic for further investigation upon her brother's positive PPRD diagnosis. At time of diagnosis, all of the patients except case 2 had manifested enlargement of the interphalangeal joints of the fingers (Figure 2a, 3a) and varying degree of limitation of joint movement, especially for the fingers and hips (Figure 2e, 2f, 3b). No signs or symptoms of inflammatory arthritis such as redness, heat, effusion, or soft tissue swelling were observed. Six patients had gait disturbance and two patients had progressed to walking difficulties that required the aid of crutches (Figure 3b) or wheels.

Laboratory investigations (table1)

The normal range of ESR and CRP were lower than $20 \mathrm{~mm} / \mathrm{hr}$ and $8 \mathrm{mg} / \mathrm{L}$, respectively. The normal range of serum level of 25-hydroxyvitamin D3 was higher than 30 ng/ml. All the patients' ESR and CRP levels were in normal range. Their liver function test, serum blood urea and creatinine, rheumatoid factor, antinuclear antibody, HLA-B27, electrolytes, thyroid function, the serum level of parathyroid hormone were all normal 
(data not shown) except for case 5 who had positive HLA-B27. After we found that case 2 had low serum level of 25-hydroxyvitamin D3, we also analyzed this parameter in case 1 , case 3 , case 6 , case 8 and case 9. The results of serum level of 25hydroxyvitamin D3 ranged from 6.16 to $22.1 \mathrm{ng} / \mathrm{ml}$, all below the normal range.

\section{Radiographic features}

All the patients' radiographs were collected and analyzed except case 7, where radiographs could not be obtained. All (8/8) patients had different degree of abnormal vertebral bodies, the severity ranging from platyspondyly and anterior blunt of the vertebral bodies (Figure 1c) to anterior beak-like of the vertebral bodies (Figure 2c). From four patients, hand X-rays were obtained which consistently showed epiphyseal enlargement of the interphalangeal joints with juxta-articular osteopenia (Figure 2b). Other radiological findings including osteopenia, reduction in hip articular space (Figure 2d), and cyst-like structures in the left femoral head (Figure 3c).

\section{CCN6 variants}

We identified 7 different $C C N 6$ variants ( 3 missense, 3 truncating, and 1 splicing) in the patients (Table 2). Case 1 and 2 had a novel homozygous variant of c.397_404del CAAGTGTT (p.Gln133Serfs*34). Case 3 and 4 had a compound heterozygous variant for c.589+2T $>$ C and c.667T $>$ G (p.Cys223Gly), which have been reported in previous cases, respectively ${ }^{16,17}$. Case 5 had a homozygous variant of c.667T $>$ G (p.Cys223Gly) ${ }^{17}$. Case 6 and 7 had a compound heterozygous variant for c.646T $>C$ (p.Trp216Arg) and c.1000T $>$ C (p.Ser334Pro) ${ }^{16,18}$. Case 8 had compound heterozygous variant of c.624dupA, (p.Cys209Metfs*21) $)^{15,19,20}$ and c.756C $>$ A (p.Cys252*) ${ }^{17}$. Case 9 had homozygous variant of $\mathrm{c} .589+2 \mathrm{~T}>\mathrm{C}^{16}$. The three missense variants were predicted to be harmful using SIFT, Polyphen-2, and CADD software. According to the American College of Medical Genetics and Genomics/the Association for Molecular Pathology variant-interpretation guidelines ${ }^{21}$, all the identified variants were classified to be pathogenic or likely pathogenic (Table 2). 


\section{Treatment and outcomes}

After obtained the definite diagnosis of PPRD, the patients were treated with physical therapy and occasional NSAIDs for pain relieve. Upon finding low serum level of 25hydroxyvitamin D3 in six patients, combined with their normal serum calcium, phosphate, alkaline phosphatase, parathyroid hormone and osteopenia, five of them had accepted treatment with oral calcitriol for 1.25 years to 1.75 years. The patients were asked to describe the severity of joint pain on a scale of $0-10$ ( 0 is no pain and 10 is severe pain), and how they are doing on daily life because of the stiffness and restricted movement of the joints on a scale of $0-10$ ( 0 is very well and 10 is very poor) before and after the treatment (table 3). After the treatment of calcitriol, case 2 proceeded without showing any symptoms, while case 6 neither improved nor worsened. The other 3 patients' joint pain, the stiffness and restricted movement of their joints were improving gradually during the treatment instead of getting worse as before. In addition, case 8 was treated with left hip arthroplasty at 32-year age and right hip arthroplasty at 35-year age. Case 7 was planning to receive hip arthroplasty.

\section{Discussion}

PPRD is a rare and inheritable skeletal dysplasia ${ }^{22}$. The clinical and radiological phenotype of patients with PPRD is rather homogeneous with progressive worsening of symptoms and radiological changes during childhood and adolescence ${ }^{2}$. The unspecific symptoms often cause a significant delay in diagnosis ${ }^{2}$, or even lead to misdiagnosis resulting in incorrect treatment ${ }^{23}$. In our nine patients with PPRD presented here, five were misdiagnosed with juvenile idiopathic arthritis (JIA) before they came to our center, and one patient (case 9) even had received treatment with immunosuppressants despite their autoimmunity markers and inflammatory parameters all within normal range. Moreover, in the two pair of sisters (case 3 and 4, case 6 and 7), when the younger sisters (case 3, case 6) were diagnosed with PPRD, their older sisters had already suffered from symptoms for 3 or 11 years, respectively. One patient (case 5) was misdiagnosed with ankylosing spondylitis only because of positive HLA- 
B27. Her parental consanguinity, normal inflammatory parameters and cyst-like structures in the left femoral head were all neglected. Most of our patients (8/9) had the onset of symptoms between 3 and 6 years of age, which is consistent with a previous review including large samples concerning age at onset ${ }^{2}$. Diagnosis of PPRD is often delayed, sometimes for decades because only few clinicians are aware of the disease ${ }^{10}$. The interval between the onset and obtaining the definite diagnosis for most of our patients (7/9) was longer than 6 years. Most of our patients presented with the characteristic manifestations of PPRD including gait abnormalities, fatigability, symmetric stiffness in multiple joints (particularly the hip), and enlarged interphalangeal joints of the hands ${ }^{10}$. Radiological features of PPRD include spondyloepiphyseal dysplasia varied from platyspondyly with loss or narrowing of intervertebral disc spaces, and anterior blunt of the vertebral bodies to anterior beaklike of the vertebral bodies, enlarged and flattened capital femoral epiphyses, short and wide femoral necks, enlarged epiphyses, loss of or narrow joint spaces in the metacarpophalangeal and interphalangeal joints and osteopenia ${ }^{2,24}$. The early radiological signs are metaphyseal enlargement of interphalangeal joints ${ }^{2}$. In our patients, we found that anterior blunt of the vertebral bodies also could be an early radiological sign when the patient (case 2) has not developed obvious clinical symptoms and characteristics yet. If the family history, clinical features presenting with abnormal gait or enlarged and stiff interphalangeal joints of the fingers but normal inflammatory markers could be combined with the characteristic radiographic findings, all the patients could get the clinical diagnosis of PPRD at very early stage of the disease. PPRD is due to a variant in Wnt1-inducible signaling protein 3 (CCN6) gene, which encodes a signaling factor involved in cartilage homeostasis and bone growth by promoting type II collagen and aggrecan expression and regulating chondrocyte proliferation and differentiation ${ }^{10}$. CCN6 expression is decreased in PPRD articular chondrocytes (ACs). PPRD ACs are characterized by increased proliferation activity and decreased apoptosis and may account for the metaphyseal enlargement seen in PPRD radiographs ${ }^{25}$. They are highly mineralized and significantly stiffer than those from normal articular cartilage which may slow down cartilage growth and repair ${ }^{11}$. All 
of our nine patients had CCN6 variants. Four patients (case 1, 2, 5, 9), including a pair of brother and sister (case 1 and 2) had homozygous variants of the CCN6 gene. Five patients (case 3, 4, 6, 7, 8) including two pairs of sisters (case 3 and 4, case6 and 7) had compound heterozygous variants of the CCN6 gene. While there is a low prevalence for PPRD in the UK, previous reports indicate it is more common in the Middle East, Gulf States, Arabic countries, and countries of the Mediterranean basin, possibly because of the high consanguinity rate in these populations ${ }^{2,3,9}$. Our case 5 had parental consanguinity and had the homozygous missense variant c.667T $>$ G, p.C223Gly in the CCN6 gene. The c.667T $>\mathrm{G}$ variant, located in exon 4 of the CCN6 gene has been reported before in Chinese and other ethnic origins' children affected with PPRD 9,23. The variant c.624dupA has been reported in patients from China, India and countries of North and West Africa ${ }^{15,19,26}$. The onset ages of patients with PPRD having homozygous variant (c.624dupA) or heterozygous (c.624dupA, c.756C $>\mathrm{A}$ ) in $C C N 6$ were much older (from 7-year old to 18-year old) than the common onset age of PPRD ${ }^{26}$. In line with this, case 8 , who presented with a compound heterozygous variant c.624dupA, c. $756 \mathrm{C}>\mathrm{A}$, had late and atypical symptoms with onset at 14 years old. Based on the information of the age of onset and genotype of the patients, we suspect that the patients with PPRD having c.624dupA variant in CCN6 may have delayed onset. One of our pair of sisters (case 6 and 7) had a compound heterozygous variant (c.646T $>C$, c. $1000 \mathrm{~T}>\mathrm{C}$ ). The variant in c.646T $>\mathrm{C}$ (p. Trp216Arg) has not been reported previously and its allele frequency was $0.00081 \%$ in gnomAD database. The missense variant c.1000T $>C$ (p.Ser334Pro) has, however, been reported in Chinese patients ${ }^{16,18}$. The patient with this compound heterozygous variant presented with marked abnormalities of cartilage chondrocytes and matrix in morphology and function. ${ }^{27}$. The other pair of brother and sister in our patients (case1 and 2) had the homozygous variant (c.397_404del CAAGTGTT) in CCN6 gene.

Although the molecular basis of PPRD has been elucidated for more than 10 years, no progress has been made towards a specific treatment ${ }^{2}$. The current treatment options only alleviate symptoms. Pain due to secondary osteoarthritis may respond to NSAIDs. Physical therapy and rehabilitation may help preserve joint mobility. Surgical 
intervention, including realignment of the lower limbs, joint arthroplasty, and/or treatment of spinal stenosis when necessary, are the mainstay of treatment today ${ }^{4,24}$. Total hip arthroplasty (THA) is a treatment option for PPRD patients to restore the joint function and improve life quality. It has been shown that THA is an effective treatment for PPRD patients with hip arthropathy, with satisfactory clinical and radiological outcome after follow-up for 18 months to 93 months (mean 47.9 months) ${ }^{20}$. Case 8 has received bilateral hip arthroplasty and since has been able to continue his job as a driver. The other adult patient is preparing to receive this treatment.

Six of our nine patients were tested for the serum level of 25-hydroxyvitamin D3 and their results were all below the normal range. Concerning their normal liver function, serum creatinine, serum calcium, phosphate, alkaline phosphatase, parathyroid hormone, we treated the patients that presented with low serum level of 25 hydroxyvitamin $\mathrm{D} 3$ with oral $1,25(\mathrm{OH})_{2} \mathrm{D}_{3}$ (calcitriol). In the follow-up appointment between 1.25 to 1.75 years we found that 2 of 5 patients' symptoms stopped progressing, while 3 of 5 patients' symptoms improved gradually. $1,25(\mathrm{OH})_{2} \mathrm{D}_{3}$ works on the key role of in calcium homeostasis and bone mineralization, which is actually the most active form of vitamin $\mathrm{D}^{28}$. It has a very short half-life and circulates in the serum at concentrations that are about $0.1 \%$ of those of the prohormone $25(\mathrm{OH}) \mathrm{D}_{3}$ and its synthesis is tightly regulated by the endocrine system. For these reasons $1,25(\mathrm{OH})_{2} \mathrm{D}_{3}$ levels in the serum are not used to evaluate the vitamin D status in humans, and $25(\mathrm{OH}) \mathrm{D}_{3}$ is favored as the best indicator of vitamin $\mathrm{D}$ status ${ }^{28}$. Recently, it has been demonstrated that $1,25(\mathrm{OH})_{2} \mathrm{D}_{3}$ accelerates chondrocyte apoptosis by impairing activation of the NF- $\kappa$ B signaling pathway in rats with rheumatoid arthritis ${ }^{13}$. The murine model also showed that $1,25(\mathrm{OH})_{2} \mathrm{D}_{3}$ controlled chondrocyte proliferation and differentiation directly ${ }^{29}$. We suspected that calcitriol may maintain the normal proliferation of articular chondrocytes by accelerating chondrocyte apoptosis ${ }^{13,14,29}$ in patients with PPRD. Therefore, our preliminary data with five patients for more than one year suggest that calcitriol may provide a potential treatment option for PPRD patients with vitamin D deficiency. However, more cases will have to be included into the study and long-term follow up is needed for a more comprehensive analysis of the 
effectiveness of calcitriol in PPRD.

\section{Conclusion}

PPRD is a rare disease characterized by progressive noninflammatory arthropathy affecting primarily the articular cartilage. Anterior blunt of the vertebral bodies could be an early radiological sign in the patient has not developed obvious clinical symptoms and characteristics yet. The patient with c.624dupA variant in CCN6 may have delayed onset of PPRD symptoms. These findings may aid in earlier diagnosis in such patients. Calcitriol, which is used in osteoporosis, could improve the clinical symptoms in the PPRD patients with vitamin D deficiency. It suggests that clinicians should seek and correct underlying vitamin D deficiency in patients with PPRD. Further studies are needed to investigate the mechanism of it.

\section{Abbreviations}

PPRD: Progressive pseudorheumatoid dysplasia

JIA: juvenile idiopathic arthritis

AC: articular cartilage

CRP: C-reactive protein

ESR: erythrocyte sedimentation rate

THA: total hip arthroplasty 


\section{Declarations}

\section{Ethics approval and consent to participate}

This study has been performed in accordance with the Declaration of Helsinki and the current ethical polices and has been approved by the Ethics Committee of the Shanghai Children's Medical Center (SCMCIRB-K2016013), Shanghai Jiao tong University School of Medicine, Shanghai, China.

\section{Consent for publication}

Written informed consents for publication were obtained from all participants.

\section{Availability of data and materials}

All the material and data of the study are available upon request.

\section{Competing interests}

The authors declare that they have no competing interests.

\section{Funding}

Not applicable

\section{Authors' contributions}

LY: designed the study, drafted the initial manuscript, wrote final manuscript, data collection, coordinated and supervised laboratory investigations, data analysis, and patient assessment. YYM: performed the literature search, conceptualized the study, collected, analyzed and interpreted the data, drafted and revised the manuscript. YFZ: communicated with the patients, collected and interpreted clinical data, and worked on manuscript. YNS: communicated with the patients, collected and interpreted clinical data, interpretation and critically reviewed the manuscript. HJC: performed the literature search, conceptualized the study, interpretation and critically reviewed the manuscript. WZ: communicated with the patients, collected and interpreted clinical 
data, and worked on manuscript. YLJ: communicated with the patients, collected and interpreted clinical data, and worked on manuscript. HH: communicated with the patients, collected and interpreted clinical data, and worked on manuscript. YGY: communicated with the patients, collected and interpreted clinical data, supervised laboratory investigations, contributed to data analysis. JW: coordinated and supervised laboratory investigations, contributed to data analysis, critically reviewed the manuscript, wrote final manuscript. All authors read and approved the final manuscript.

\section{Acknowledgements}

We thank Dr. Emely Verweyen (Cincinnati Children's Hospital Medical Center, Ohio, U.S.) for revising the paper sentence by sentence.

\section{References}

1. Wynne-Davies R, Hall C, Ansell BM. Spondylo-epiphysial dysplasia tarda with progressive arthropathy. A "new" disorder of autosomal recessive inheritance. The Journal of bone and joint surgery. British volume. 1982;64(4):442-445.

2. Garcia Segarra N, Mittaz L, Campos-Xavier AB, et al. The diagnostic challenge of progressive pseudorheumatoid dysplasia (PPRD): a review of clinical features, radiographic features, and WISP3 mutations in 63 affected individuals. American journal of medical genetics. Part C, Seminars in medical genetics. 2012;160C(3):217-229.

3. Pomeranz CB, Reid JR. Progressive pseudorheumatoid dysplasia: a report of three cases and a review of radiographic and magnetic resonance imaging findings. Skeletal radiology. 2019;48(9):1323-1328.

4. Giray E, Yagci I, Elcioglu HN. Progressive pseudorheumotoid dysplasia: A presentation of four cases with slow and rapid progression and effects of early rehabilitation program. Turkish journal of physical medicine and rehabilitation. 2019;65(3):290-297. 
5. Chen W, Mo S, Luo G, et al. Progressive pseudorheumatoid dysplasia with newfound gene mutation of Wntl inducible signaling pathway protein 3. Pediatric rheumatology online journal. 2018;16(1):55.

6. Fathalla BM, Elgabaly EA, Tayoun AA. Coexistence of a novel WISP3 pathogenic variant and an MEFV mutation in an Arabic family with progressive pseudorheumatoid dysplasia mimicking polyarticular juvenile idiopathic arthritis. Pediatric rheumatology online journal. 2020;18(1):69.

7. Neerinckx B, Thues C, Wouters C, Lechner S, Westhovens R, Van Esch H. A homozygous deletion of exon 1 in WISP3 causes progressive pseudorheumatoid dysplasia in two siblings. Human genome variation. 2015;2:15049.

8. Pode-Shakked B, Vivante A, Barel O, et al. Progressive Pseudorheumatoid Dysplasia resolved by whole exome sequencing: a novel mutation in WISP3 and review of the literature. BMC medical genetics. 2019;20(1):53.

9. Sailani MR, Chappell J, Jingga I, et al. WISP3 mutation associated with pseudorheumatoid dysplasia. Cold Spring Harbor molecular case studies. $2018 ; 4(1)$.

10. Torreggiani S, Torcoletti M, Campos-Xavier B, et al. Progressive pseudorheumatoid dysplasia: a rare childhood disease. Rheumatology international. 2019;39(3):441-452.

11. Lin LJ, Ge YM, Tian Y, et al. Multi-scale mechanical investigation of articular cartilage suffered progressive pseudorheumatoid dysplasia. Clinical biomechanics. 2020;79:104947

12. Lung BE, Komatsu DEE. Calcitriol. StatPearls. Treasure Island (FL)2020.

13. Tian R, Li X, Li Y, Wang $\mathrm{K}$, Wang C, Yang P. 1,25(OH)2D3 promotes chondrocyte apoptosis and restores physical function in rheumatoid arthritis through the NF-kappaB signal pathway. Biomedicine \& pharmacotherapy $=$ Biomedecine \& pharmacotherapie. 2018;106:149-155.

14. Avcioglu G, Ozbek Iptec B, Akcan G, et al. Effects of 1,25-Dihydroxy vitamin D3 on TNF-alpha induced inflammation in human chondrocytes and SW1353 cells: a possible role for toll-like receptors. Molecular and cellular biochemistry. 
2020;464(1-2):131-142.

15. Ye J, Zhang HW, Qiu WJ, et al. Patients with progressive pseudorheumatoid dysplasia: from clinical diagnosis to molecular studies. Molecular medicine reports. 2012;5(1):190-195.

16. Sun J, Xia W, He S, et al. Novel and recurrent mutations of WISP3 in two Chinese families with progressive pseudorheumatoid dysplasia. PloS one. 2012;7(6):e38643.

17. Luo H, Shi C, Mao C, et al. A novel compound WISP3 mutation in a Chinese family with progressive pseudorheumatoid dysplasia. Gene. 2015;564(1):35-38.

18. Sertedaki A, Papadimitriou A, Voutetakis A, Dracopoulou A, Maniati-Christidi M, Dacou-Voutetakis C. Gene symbol: TSH B. Disease: congenital hypothyroidism. Human genetics. 2004;115(2):174.

19. Bhavani GS, Shah $\mathrm{H}$, Dalal AB, et al. Novel and recurrent mutations in WISP3 and an atypical phenotype. American journal of medical genetics. Part A. 2015;167A(10):2481-2484.

20. Feng B, Xiao K, Ren Y, et al. Mid-Term Outcome of Total Hip Arthroplasty in Patients With Progressive Pseudorheumatoid Dysplasia. Journal of clinical rheumatology : practical reports on rheumatic \& musculoskeletal diseases. 2019; doi:10.1097/RHU.0000000000001248

21. Richards S, Aziz N, Bale S, et al. Standards and guidelines for the interpretation of sequence variants: a joint consensus recommendation of the American College of Medical Genetics and Genomics and the Association for Molecular Pathology. Genetics in medicine : official journal of the American College of Medical Genetics. 2015;17(5):405-424.

22. Hu Q, Liu J, Wang Y, et al. Delayed-onset of progressive pseudorheumatoid dysplasia in a Chinese adult with a novel compound WISP3 mutation: a case report. BMC medical genetics. 2017;18(1):149.

23. Al Kaissi A, Kenis V, Jemaa LB, et al. Skeletal phenotype/genotype in progressive pseudorheumatoid chondrodysplasia. Clinical rheumatology. 2020;39(2):553-560. 
24. Bhavani GSL, Shah H, Shukla A, Dalal A, Girisha KM. Progressive Pseudorheumatoid Dysplasia. In: Adam MP, Ardinger HH, Pagon RA, et al., eds. GeneReviews((R)). Seattle (WA)2015.

25. Zhou $\mathrm{HD}, \mathrm{Bu} \mathrm{YH}$, Peng $\mathrm{YQ}$, et al. Cellular and molecular responses in progressive pseudorheumatoid dysplasia articular cartilage associated with compound heterozygous WISP3 gene mutation. Journal of molecular medicine. 2007;85(9):985-996.

26. Chouery E CS, Dahmen J, Zouari L, Gribaa M, Leban N, Chibani B. Progressive pseudorheumatoid dysplasia in North and West Africa: clinical description in ten patients with mutations of WISP3. Egypt J Med Hum Genet. 2017;18(3):299-303.

27. Peng YQ, Liao EY, Gu HM, et al. [Pathology and molecular pathogenesis of spondyloepiphyseal dysplasia tarda with progressive arthropathy caused by compound CCN6 heterogeneous gene mutations]. Zhonghua yi xue za zhi. 2004;84(21):1796-1803.

28. Brandi ML. Indications on the use of vitamin D and vitamin D metabolites in clinical phenotypes. Clinical cases in mineral and bone metabolism : the official journal of the Italian Society of Osteoporosis, Mineral Metabolism, and Skeletal Diseases. 2010;7(3):243-250.

29. Hirota Y, Nakagawa K, Mimatsu S, et al. Nongenomic effects of 1alpha,25dihydroxyvitamin D3 on cartilage formation deduced from comparisons between Cyp27b1 and Vdr knockout mice. Biochemical and biophysical research communications. 2017;483(1):359-365. 


\section{Figure Legends}

Figure 1. Images of case 2 (girl, 4 years old). a. Normal fingers b. Normal squatting

c. Radiograph of the thoracolumbar spine showing anterior blunt of the vertebral bodies $\mathbf{d}$. normal radiograph of pelvis

Figure 2. Images of case 1 (boy, 9 years old). a. Enlarged interphalangeal joints of the fingers b. Radiograph of left hand showing epiphyseal enlargement of the interphalangeal joints with juxta-articular osteopenia c. Radiograph of the thoracolumbar spine showing platyspondyly and anterior beak-like of the vertebral bodies $\mathbf{d}$. Radiograph of pelvis showing osteopenia, reduction in hip articular space e. Photograph of both hands at 9 years old f. Photograph of both hands at 16 years old Figure 3. Images of case 3 (girl, 13 years). a. Enlarged interphalangeal joints of the fingers b. Enlarged elbows, walking difficulty and requiring crutches $\mathbf{c}$. Radiograph of pelvis showing osteopenia, reduction in hip articular space, and cyst-like structures in the left femoral head 


\section{Figures}
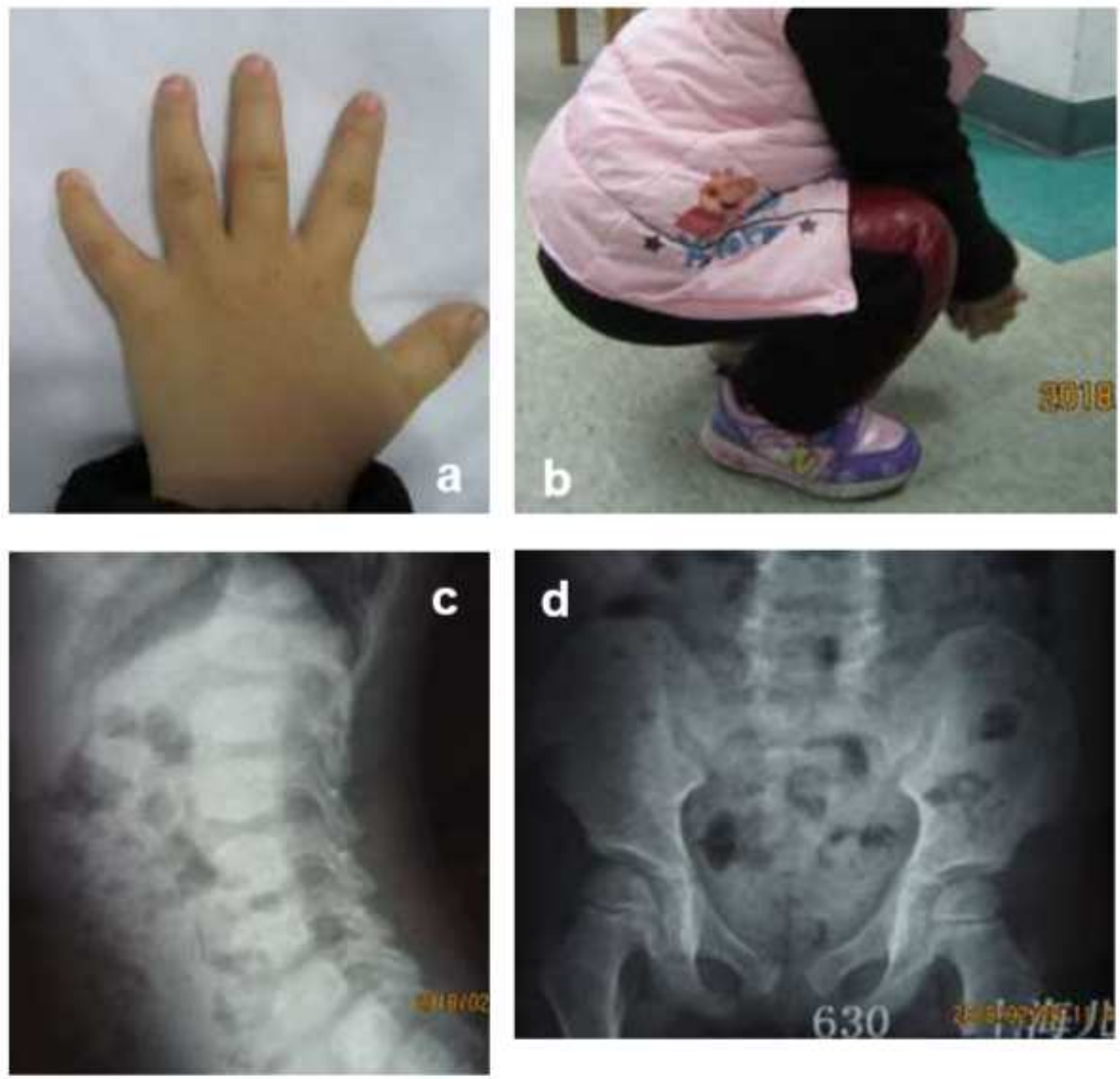

\section{Figure 1}

Images of case 2 (girl, 4 years) a. Normal fingers b. Normal squatting c. Radiograph of the thoracolumbar spine showing anterior blunt of the vertebral bodies d. normal radiograph of pelvis 

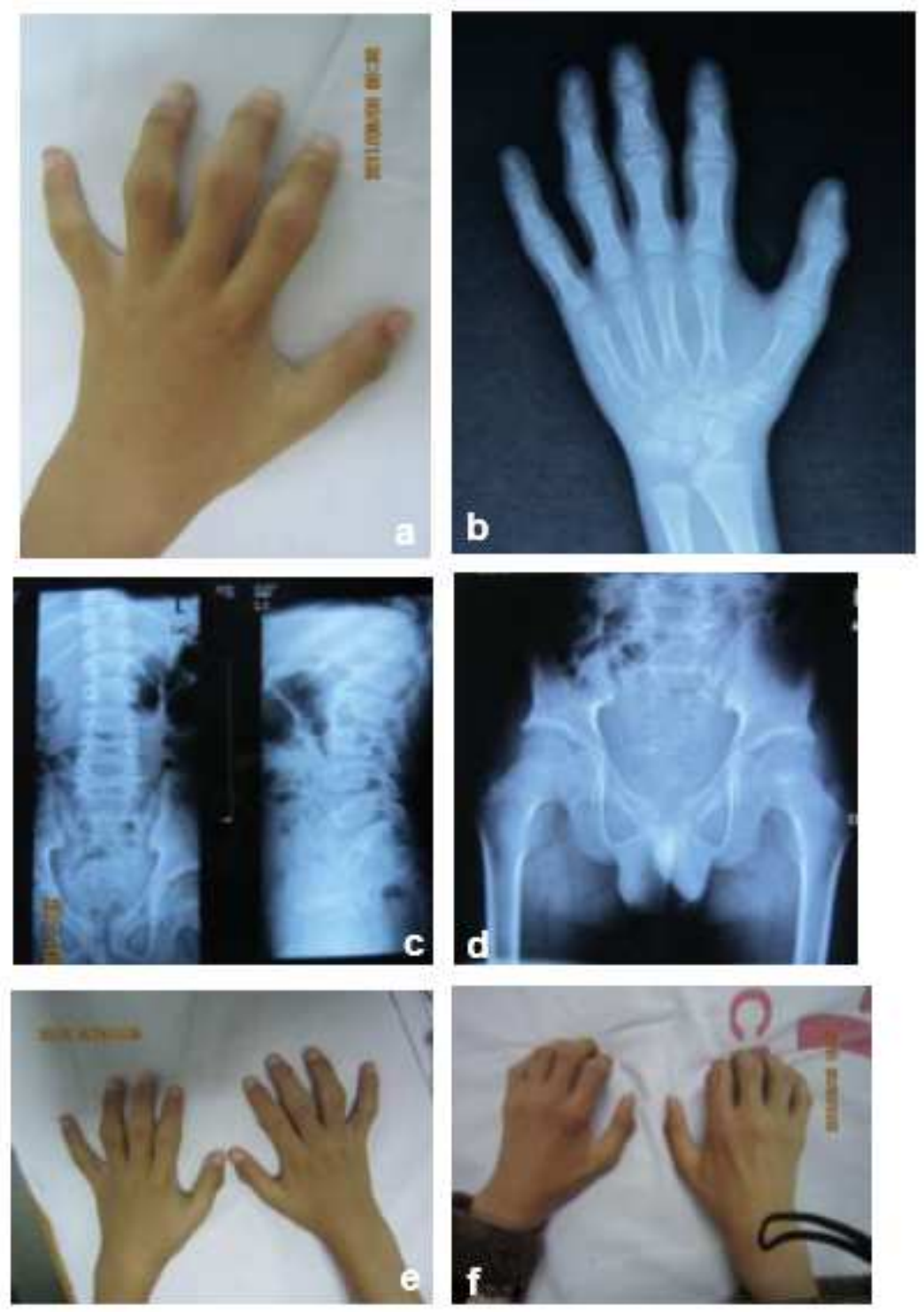

Figure 2

Images of case 1 (boy, 9 years) a. Enlarged interphalangeal joints of the fingers $b$. Radiograph of left hand showing epiphyseal enlargement of the interphalangeal joints with juxta-articular osteopenia $\mathrm{c}$. Radiograph of the thoracolumbar spine showing platyspondyly and anterior beak-like of the vertebral bodies $d$. Radiograph of pelvis showing osteopenia, reduction in hip articular space e. Photograph of both hands at 9 years old $f$. Photograph of both hands at 16 years old 

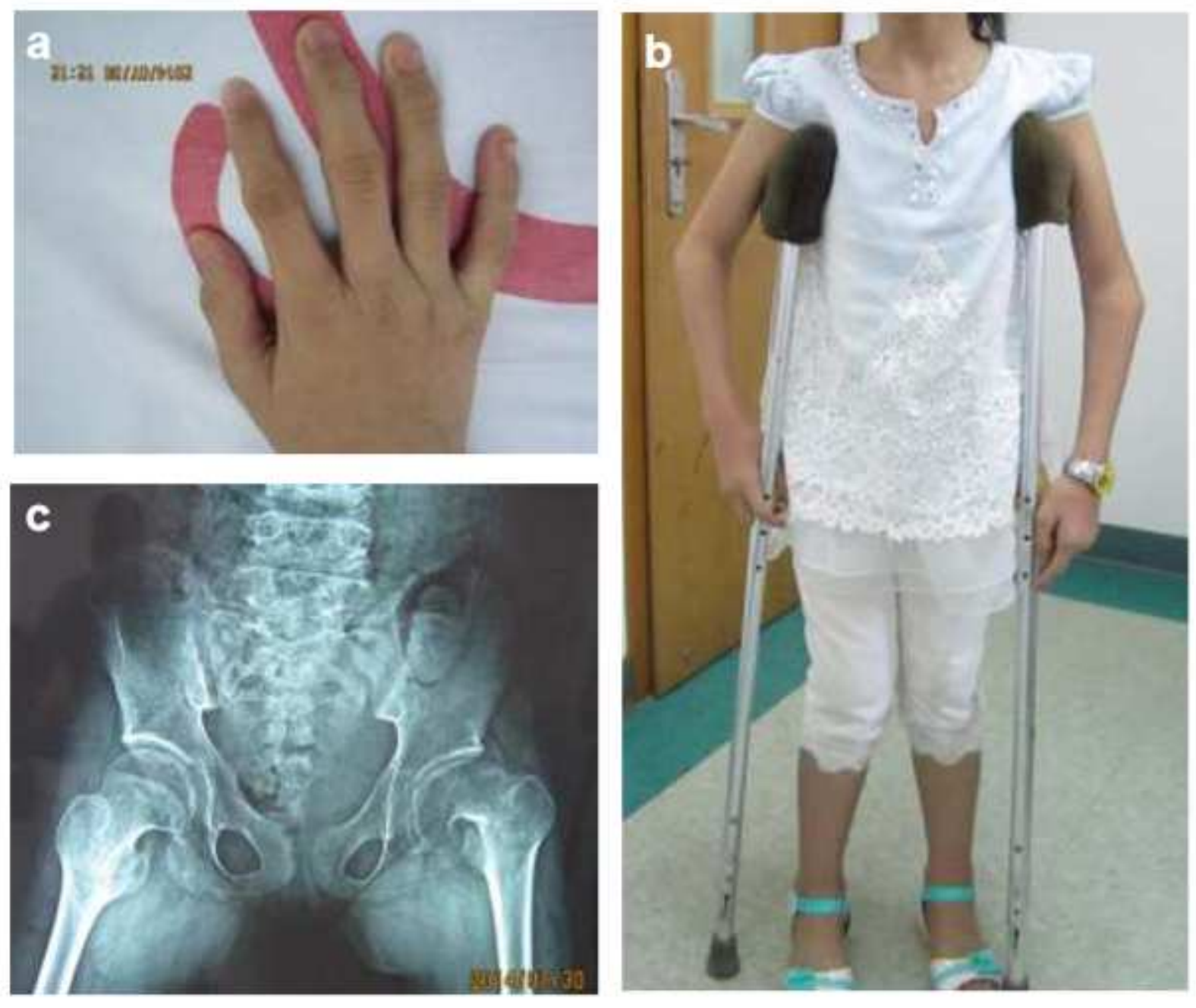

\section{Figure 3}

Images of case 3 (girl, 13 years) a. Enlarged interphalangeal joints of the fingers b. Enlarged elbows, walking difficulty and requiring crutches $\mathrm{c}$. Radiograph of pelvis showing osteopenia, reduction in hip articular space, and cyst-like structures in the left femoral head

\section{Supplementary Files}

This is a list of supplementary files associated with this preprint. Click to download.

- 2021215table.pdf 